\title{
EDTA and kKCI Doped KDP Solution and Thermodynamics Principle of Crystallization Process
}

\author{
Dehong Li,Ruifang Bai,Zhangang Huo \\ School of Vehicle and Power Engineering, Jiuquan Science and Technology University, Gansu, China
}

\begin{abstract}
Potassium dihydrogen phosphate, KH2PO4 (KDP) crystal is an excellent electro-optical nonlinear optical crystal with large electro-optical nonlinear coefficient, high laser damage threshold, and laser frequency doubling effect, electro-optical effect, Piezoelectric effects and other special features, widely used in inertial confinement fusion engineering (ICF) and electro-optical switching devices. Therefore, its growth mechanism, growth process and performance have been systematically studied. In the process of KDP crystal growth, it is found that the stability of the growth solution is an important factor affecting the quality of crystal growth. Therefore, in recent years, more and more research on the stability of the solution, such as the study of ph, doping, supersaturation, overheating time on the stability of the solution. Among them, the research on the doping is mostly reported, and the research on this aspect is mainly focused on two aspects. On the one hand, it is the study of the stability of the solution under doping, and the other is the effect of doping on the optical quality of the crystal. In fact, the stability of the growth solution and the quality of crystal growth is directly related to the quality, but the existing research to isolate the two researches. Therefore, the experiment will be carried out in the case of double-doped KDP solution stability, KDP crystal growth and crystal optical quality and other experiments, and in-depth analysis of the impact of solution stability and crystal optical quality of the reasons, while the solution stability and The relationship between the optical quality of the crystal is briefly analyzed.
\end{abstract}

Key words:KDP crystal, double doping, stability, nucleation, dislocation density

\section{1、 Introduction}

\subsection{Research topics and significance}

The 21 st century has a rapid development of high-tech era, such as new materials technology, information technology, new energy technology developments has swept the world, and are also widely used. How can the development of these technologies a good embodiment of a country's comprehensive national strength, therefore, with high-tech industry advantage is one of the important signs of national development. And the rapid development of new materials is the development of high-tech industry is an important aspect. The National Eleventh Five-Year Plan also clearly pointed out: to accelerate the development of high-tech industries, emphasizing the development of new materials industry, and focus on the development of special functional materials and other industrial groups, establish and improve the new material innovation system.

New materials can be divided into functional materials and structural materials by use. The functional materials are those which have excellent electrical, optical, thermal, mechanical and other functions, and can complete the transformation between functions for the manufacture of various functional components (Such as KDP used in laser frequency conversion, electro-optical modulation, acoustooptic modulation, electro-optical Q-switched lasers, parametric oscillator, piezoelectric transducers and optical fast switch) and is widely used in various high-tech areas of high-tech materials; When focusing on the use of its mechanical properties of the material known as the structural material.

So far, there have been many studies on phosphate crystals (such as KDP, DKDP and ADP) in functional materials. Such crystal materials have been widely used in inertial confinement fusion engineering (ICF) and electro-optical devices. However, it is not so easy to grow crystals that meet the engineering requirements. Nearly 70 years, some scholars have done a lot of research on how to optimize the crystal growth, but also get a lot of results. Therefore, based on the existing theoretical basis of crystal growth and related experimental results, this subject studies the effect of doping on the stability of KDP crystal growth solution and its growth dislocation from doping.

$1.2 \mathrm{KDP}$ crystal structure
In the period from 1925 to 1955 , West et al. $[1,2]$ studied the internal structure of KDP crystal under different experimental conditions by X-ray and neutron diffraction techniques, and proposed some structural parameters of KDP crystal. KDP crystal at a certain temperature $(123 \mathrm{~K})$ above belongs to the tetragonal system, the point group, the space group. The unit cell parameters $\mathrm{a}=\mathrm{b}=7.4528 \mathrm{~nm}, \mathrm{c}=$ $6.9717 \mathrm{~nm}$, each unit contains four primers. The ideal shape of the crystal is a tetragonal column and a tetragonal double cone of the polymer. In the KDP crystal, there is a strong polarization between the $\mathrm{P}$ atom and the $\mathrm{O}$ atom, and the $\mathrm{PO} 4$ tetrahedron is formed by the covalent bond. Each $\mathrm{P}$ atom is surrounded by four $\mathrm{O}$ atoms at the approximate tetrahedron angle: $\mathrm{P}$ the atoms and the $\mathrm{K}$ atoms are arranged alternately in the C-axis direction at c / 2 intervals. Each PO4 group is connected with the other four PO4 tetrahedra with hydrogen bonds adjacent to and adjacent to the $\mathrm{C}$-axis direction, the group is connected not only to the hydrogen-keyboard system, but also to the $\mathrm{K}$ atoms. There are eight adjacent $\mathrm{O}$ atoms around each $\mathrm{K}$ atom. These adjacent atoms, O, can be divided two interspersed into two PO4 tetrahedron, KDP crystal structure model diagram had shown in Figure 1.1 .

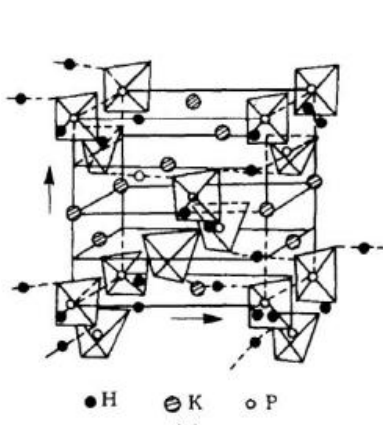

(a)

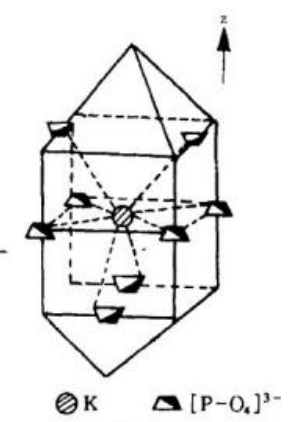

(b)
Fig. 1.1 The structure model of the KDP crystal and ideal shape of KDP crystal

1956 P.Hartman ${ }^{[3]}$ used the PBC (periodic bond chain) theory to predict the ideal shape of the KDP crystal. It is found that there are four strong PBC key chains in the KDP crystal: A: [001] direction, that is, $\mathrm{P}(000)-\mathrm{K}\left(00 \frac{1}{2}\right)-\mathrm{P}(001)$ key chain, this key chain is linear, 
key length $0.3486 \mathrm{~nm}$; $\mathrm{B}:(100)$ direction, that is, $\mathrm{P}(000)-\mathrm{K}$ $\left(\begin{array}{lll}\frac{1}{2} & 0 & \frac{1}{4}\end{array}\right)-\mathrm{P}(100)$ key chain, this key chain is a broken line type, the key length is $0.4114 \mathrm{~nm} ; \mathrm{C}:\left[\frac{1}{2} \frac{1}{2} \frac{1}{2}\right.$ ] direction, that is, $\mathrm{P}$ ( 000$)-\mathrm{K}\left(\frac{1}{2} \quad 0 \frac{1}{4}\right)-\mathrm{P}\left(\frac{1}{2} \frac{1}{2} \frac{1}{2}\right)$ key chain, this key chain for the fold line, the keyboard length $0.4114 \mathrm{~nm}$; the other for the weak along the [110] direction of the D key chain (key length $0.527 \mathrm{~nm}$ ), this key because of relatively weak, So in the prediction of crystal open when not consider. The above four pbcs can divide the crystal into five flat surfaces: $\{010\}\{011\}\{001\}\{110\}\{112\}$.

\section{2、Thermodynamics principle of crystallization process}

The formation of new phase, including crystal nucleation and crystal growth of the two processes, called 'nuclear' and 'crystallization'. When the maternal phase is in equilibrium with the new phase, some atoms or molecules gather to form germs because some local regions of the thermal fluctuation (or fluctuation) system always deviate from the equilibrium state (density fluctuation). At the same time these atoms or molecules may be separated, to restore the original state [39]. Only supersaturation is further increased, so that the solution system to the unstable saturation zone, nucleation in the solution in the spontaneous. If the solution is crystallized, there must be a driving force in the phase change process to destroy the metastable state, so that the phase change can be carried out in the direction of crystallization. However, the driving force is dependent on the free energy. The formation of the nuclei is the combined effect of the two processes: on the one hand, the gas or liquid in the metastable state, tends to agglomerate on the surface of the Gibbs function spontaneously And on the other hand is the process of increasing the surface Gibbs function by increasing the surface area of the condensed state. The new phase is formed by the condition that the system's Gibbs function is minimal, i.e. $\Delta G=\Delta G_{V}+\Delta G_{S}$ The smallest, which $\Delta G_{V}$ is the system free energy changes, $\Delta G_{S}$ the surface free energy changes, which can be the formation of new phase conditions $^{[40]}$, which is the principle of crystallization thermodynamics.

Crystallization dynamics equations and parameters play an important role in the analysis, design and operation of industrial crystallization processes. In general, crystallization kinetics includes a wide range of contents such as nucleation kinetics, growth kinetics, crystallization molecular dynamics, and fluid dynamics. It is generally believed that crystallization kinetics include only nucleation and growth kinetics.

The crystalline particles (new phase) formed in the supersaturated solution are called nuclei. The formation of nuclei can be divided into two categories:

Primary nucleation: nucleation in the absence of crystals in solution systems;

Secondary nucleation: nucleation in the presence of crystals in solution systems.

Specific nuclei formation method as shown in Figure 2-1 ${ }^{[41]}$ :

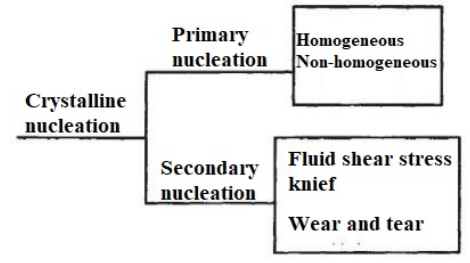

Fig 2.1 The diagram of the conformation of nuclei

2.1 Primary nucleation

Primary nucleation is divided into homogeneous primary nuclei (Nucleation) and heterogeneous nucleation (Heterogeneous primary Nucleation) two kinds of uniform nucleation is spontaneous generation, rather than by the external particles or substrate induced; and nonuniform the nucleus is produced by the induction of foreign particles or substrates.

2.1.1 The driving force of the new phase formation ${ }^{[40]}$

The formation of the new phase requires a certain system of 'over-saturated' or 'cold' and other conditions. For example, in the solution, the solute crystallization process requires that the solution system must be in a supersaturated state (i.e., the solute concentration in the solution exceeds the solution saturation concentration), so that it is possible to crystallize in the solution. However, even if the solution system to maintain a certain degree of supersaturation, it may not occur crystal phase transition process. We know that the supersaturation of the solution system can be maintained at a certain time is the reason for the existence of surface effects, therefore, only to the new phase of a driving force, so as to achieve the crystal phase transition process. Under certain pressure conditions, the new phase formed Gibbs function changes. Such as the condition of melt is also known as.

$$
G=H-T S
$$

When the two phases are balanced, $G_{L}=G_{S}$ which, respectively $\mathrm{L}, \mathrm{S}$ represent the melt and the crystal, there are

$$
\begin{aligned}
& \qquad H_{L}-T S_{S}=H_{S}-T S_{S} \\
& \text { and } \Delta G=G_{L}-G_{S} \\
& \text { then } \Delta H=T \Delta S \\
& \qquad T_{C} \text { under the melting point, there are } \Delta S_{C}=\Delta H / T_{C}
\end{aligned}
$$

For the temperature near the melting point $\mathrm{T}$, the $\Delta S_{C}$ entropy change can be expressed as, and then the two-phase interface of the guise changes to

$$
\begin{aligned}
& \Delta G=\Delta H-T \Delta S_{C}=\Delta H-T \frac{\Delta H}{T_{C}}=\Delta H\left(1-\frac{T}{T_{C}}\right) \\
& \Delta T=T_{C}-T \text { Introductionis called 'undercooling', has } \\
& \Delta G=\Delta H \frac{\Delta T}{T_{C}}
\end{aligned}
$$

also known $\Delta H$ as phase change latent heat, for a given substance, with a fixed value.

When the vapor phase is condensed into droplets in the vapor phase, the new phase driving force is related to the saturated vapor pressure $P_{2}$, and the saturated vapor pressure of the vapor phase and the saturated vapor pressure of the metastable state are $P_{1}$, and the driving force of the new phase is dependent on the vapor saturation, 


$$
\Delta G=\frac{k}{v} \operatorname{TIn} \frac{P_{1}}{P_{2}}
$$

If the crystals are crystallized in solution, the driving force of the new phase is related to the solute in the solution. The supersaturation concentration of the metastable state of the solution is, that the saturated concentration is, the concentration expression of the new phase formed by the thermodynamic principle,

$$
\Delta G=\frac{k}{v} \operatorname{TIn} \frac{C_{1}}{C_{2}}
$$

\subsubsection{Uniform nucleation}

Uniform nucleation is an ideal situation, you can use the thermodynamic theory to calculate the theory. In the process of uniform nucleation, the formation of a new crystal phase particle, caused by the Gibbs free energy change by the two processes to determine the molecular binding process into a crystal embryo and the formation of new crystal phase particles.

$$
\Delta G=\Delta G_{S}+\Delta G_{V}=4 \pi r^{2} \gamma-\frac{4}{3} \pi r^{3} \Delta G_{V}
$$

Which is the crystal embryo radius, is the nucleus surface area per unit area of the surface energy (i.e. solid-liquid interface tension). at room temperature and pressure, it is a function of temperature and increases with increasing temperature.

Where,

$$
\Delta G_{V}=\frac{k}{\Omega_{s}} T \operatorname{In} \frac{C_{1}}{C_{2}}=\frac{4 \pi r^{3} \Delta \mu}{3 \Omega_{s}}
$$

The driving force for the formation of the new phase is the work of forming the $\mathrm{r}$ radius of the nucleus, that is, the volume $\Omega_{s}$ of free energy. $C_{1}$ In the formula, $C_{2}$ the volume of a molecule of nucleation grain is the actual concentration of the solution, $\Delta \mu$ which is the saturation concentration $r$ of the solution, which is the change of the chemical potential during the formation of the crystal embryo.

When the formation of nuclei in the process of dissolution and accumulation to achieve equilibrium, there are:

$$
\frac{d \Delta G}{d r}=0
$$

Get nuclear conditions:

$$
2 \gamma-r \Delta G_{V}=0
$$

The critical radius of the nuclei can be obtained:

$$
r^{*}=\frac{2 \gamma}{\Delta G_{V}}=\frac{2 \gamma \Omega_{s}}{\Delta \mu}
$$

Equation (2.3) determines the balance between the crystal embryo and the surrounding matter. When the size of the crystal embryo is less than that, the free energy decreases with the decrease of the size of the crystal embryo, so the crystal embryo will eventually dissolve. When the crystal embryo size is larger than that, the free energy decreases with the increase of the crystal embryo size, so the crystal embryo will grow bigger. To form nuclei that reach a critical nucleation radius (), a system should overcome the energy barrier (critical nucleation work), i.e., the nucleation barrier, which can be expressed as:

$$
\Delta G^{*}=\frac{16}{3} \pi \gamma^{3} / \Delta G_{V}^{2}
$$

Molecules only through the barrier, to reach the critical radius will be nucleated in order to spontaneous crystallization growth, as shown in Figure 2.2 ${ }^{[42]}$

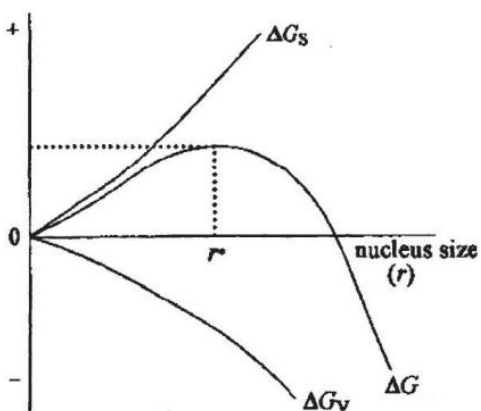

Figure 2.2 Relationship between critical nucleation and surface free energy

Fig. 2.2 The relationship of critical nucleation work and surface free energy

\subsubsection{No uniform nucleation}

In the crystal growth system, many external factors (wall, liquid surface environment, etc.) Will affect the nucleation of the solution system, therefore, most nucleation belongs to the non-homogeneous nucleation process. We discuss the nucleation conditions at the interface of heterogeneous media. The nucleation is carried out at a solid-liquid interface where the liquid moistens the solid surface and nucleates at the solid interface, as shown in Figure 2-3 [40]. The radius of the nuclei in the surface of the solid surface is the radius of the nuclei. The interface energy of the solid-liquid interface is the interface energy change in the liquid phase during the no uniform nucleation process. The interface energy of the liquid-liquid interface is the change of the surface area of the crystal nucleus in the liquid phase. The surface energy of the core-solid interface is the amount of nucleation contact area grown on the solid surface.

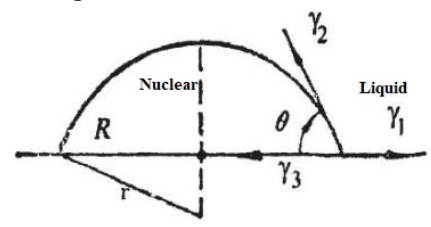

Figure 2.3 Schematic diagram of wet nucleation

Fig 2.3 Schematic diagram of wet nucleation

In the nucleation process, due to the interface caused by the function Gibbs change, for no uniform nucleation,

$$
G_{S}^{*}=G_{A 1}^{*}+G_{A 2}^{*}+G_{A 3}^{*}=\gamma_{1} A_{1}^{*}+\gamma_{2} A_{2}^{*}+\gamma_{3} A_{3}^{*}
$$

When the nucleus is formed, the solid-liquid interface reduction should be equal to the area of nuclear solid-liquid interface,

$$
A_{3}^{*}=-A_{1}^{*}=\pi R^{2}
$$

The core-liquid interface is the corresponding spherical crown area, which can be obtained from the three-dimensional geometry

$$
\begin{aligned}
& A_{2}^{*}=2 \pi r^{2}(1-\cos \theta) \\
& \text { Then } \quad G_{S}^{*} 2 \pi r^{2}(1-\cos \theta)+\pi R^{2}\left(\gamma_{1}-\gamma_{3}\right)
\end{aligned}
$$

To represent the nucleus volume, the crown volume formula is available

$$
V^{*}=\pi r^{3}\left(\frac{2-3 \cos \theta+\cos ^{2} \theta}{3}\right)
$$

In the no uniform nucleation, the total Gibbs free energy of the 
system changes

$$
G^{*}=G_{S}^{*}+G_{V}^{*}=G_{S}^{*}+V^{*} \Delta G_{V}
$$

To achieve a balance, $\frac{\partial G^{*}}{\partial r}=0$

Can be obtained crystal nucleus critical radius $r_{C}^{*}=\frac{2 \gamma_{2}}{\Delta G_{V}}$

\subsection{Secondary nucleation}

The solution system in the presence of solid phase crystals at nucleation is known as secondary nucleation. On the one hand, secondary nucleation is a multiphase nucleus, which is carried out in a multiphase system. On the other hand, the appearance of the crystallization center in the secondary nucleation process is, in many cases, indistinguishable from the general homogeneous nucleation in principle. This refers to the generation of new particles in the solid phase of the solution body due to the presence of seed crystals.

The mechanism of secondary nucleation is complicated and has not yet been studied. Therefore, the source of secondary nucleation and the mechanism of its formation have been the subject of scientific research among scientists. At present, this study is still in the semiempirical semi-theoretical stage. The secondary nucleation mechanism can be divided into two groups [43].

The first category is various forms of non-contact nucleation. Non-contact nucleation is the case where the new nuclei appear in the solution in the case where the solid phase is not in direct contact, that is, the crystals do not come into contact with or collide with each other or with the crystallizer wall or other equipment. For example, when a new crystal center is generated when a crystal or a few crystals are added to the solution, if the crystal is added to a container containing a supersaturated solution and the crystal is free to drop to the bottom, it is often seen that There is a new crystal cloud in the process of crystal settling [43], followed by particles. It is believed that the new crystal cloud is formed by the microcrystals located on the crystal surface. When the crystals precipitate in the solution, the microcrystals are washed down and begin to grow up alone.

Crystals grown at larger supersaturation form fine needle-like crystals and dendritic crystals, and the solution flows to destroy these crystals and the resulting grains become new crystal centers. That is, the seed surface in the interaction with the flow of local damage under the mechanism. The correctness of this mechanism has been demonstrated for the data of magnesium sulfate crystals [44].

The second category is contact nucleation. The contact nucleation is directly related to the collision, the surface to the surface of the sliding, friction and other factors directly related. The mechanism of the analysis there are many, one of the most simple is the crystal collision or collision with the equipment surface is broken. Each as the broken particles becomes independent of the crystallization center. The number of secondary nuclei obtained according to the contact mechanism depends on the supersaturation of the solution, the contact area of the solid particles, the energy of the interaction and the nature of the crystalline material.

A portion of the data for the second nucleation can be expressed by the following equation ${ }^{[45]}$

$$
J / A=k_{1} \delta E_{\text {imp }} A^{-\gamma_{2}} \text { versus } J / A=k_{2} \delta E_{\text {imp }}
$$

Where $\mathrm{J}$ - the number of nuclei due to a collision;

A- The contact area at the time of the collision;

$\delta$ - Relative supersaturation;

$$
E_{\text {imp }} \text { - Collision energy. }
$$

The first formula is the 'crystal-device surface' (mold wall stirrer, etc.) Collide with each other, the second formula that 'crystal-crystal' collide with each other. The secondary nucleation rate according to the contact mechanism can be calculated as follows:

$$
d J=k_{3}(\Delta c)^{P} \omega d_{P} E_{\text {imp }} n_{0}\left(d_{P}\right)
$$

In the above equation, the absolute supersaturation index $\mathrm{P}$ is an empirical value, which is the crystal size and is the rotational frequency. For a particular system, the solution of the above equation has a different form. For example, for 'crystal-crystal' interaction, in the mixing mold is:

$$
J=k(\Delta c)^{P} E m_{\min }^{2}
$$

The collision in the above system is due to agitation, since it determines the velocity of the motion and the mass of the heavy particles of the suspension as the mass of the smallest size particles sufficient to produce the nuclei. $m_{\min }$ Although the secondary nucleation has various characteristics, but is the principle of homogeneous nucleation as the basis, this foundation and other types of nucleation the same.

\subsection{Nucleation rate}

The nucleation rate is the number of nuclei formed in the unit volume per unit time. In discussing $\mathrm{J}$ phase transitions, the terms of nuclei and crystal centers are generally regarded as the same concept and refer to the growing crystals. Crystalline center is, in essence, a new phase of particles that can continue to grow, or other solid particles that can be used as raw materials. The nucleation rate of the research method is in fact the number of crystallization centers.

Nucleation rate is controlled by two factors: one is the probability of formation of nuclear embryos during phase change, and one is the probability of molecular transition to nuclear embryo in the process of diffusion.

The nucleation rate per unit volume is

$$
J=\frac{D}{r^{* 2}} n \exp \left[-\Delta G_{\max } / k T\right.
$$

Substituting (2.4) into (2.5)

$$
J=\frac{D}{a^{2}} n \exp \left[-\frac{k \gamma_{s}^{3} v^{2}}{(k T)^{3}(\operatorname{InS})^{2}}\right]
$$

Among them $\ln S=\ln \frac{C_{1}}{C_{2}}$, the diffusion coefficient of the particles migrating from the liquid phase to the surface of the crystal embryo

$$
D=D_{0} \exp \left(-\frac{\Delta G_{\max }}{R T}\right)=k T / 6 \pi \eta r
$$

Approximate calculation (is the viscosity coefficient of the medium, is the particle radius), is the average particle spacing. Formula (2.7) associates the nucleation rate with the temperature supersaturation coefficient and the physical properties of the crystalline material. In addition, the rate of crystallization centers is also related to temperature, crystallization of hydrodynamic conditions, various mechanical effects, electric and magnetic fields. The influence of various factors on nucleation rate is qualitative.

Factors that affect the rate of nucleation are as follows:

1) Supersaturation ratio

The nucleation rate is largely dependent on the degree of supersaturation, and the number of particles formed in the new phase should increase sharply as it increases and tend to zero as the decrease 
decreases. At some limit supersaturation, the nucleation rate is equal to zero. The relationship between nucleation rate and supersaturation ratio is shown in Figure 2.4 $4^{[43]}$.

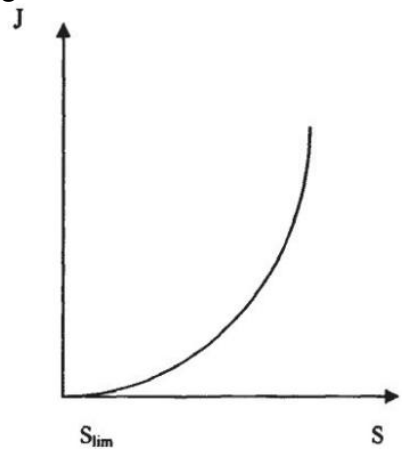

Figure 2.4 The relationship between nucleation rate and supersaturation ratio

Fig. 2.4 Relation between nucleation rate and supersaturation ratio

\section{2) Temperature}

The relationship between temperature and nucleation rate is more complex and is interdependent with other crystallization parameters. For example, changes in temperature can cause solution viscosity, particle kinetic energy, solvent structure, solubility changes. So discuss the temperature must take into account the characteristics of the process associated with this change. According to the effect of the above temperature pairs will increase the nucleation rate with the increase. Of course, the premise is that the other values are independent of the above.

\section{3) Mechanical action}

Mechanical effects have a significant effect on the appearance of nuclei. Such as vibration, in the supersaturated solution occurred in the surface of each other impact, stirring. Often the slight vibration of the supersaturated solution or the addition of a solid to the solution will begin to produce nuclei in the solution. In the absence of seed, mechanical vibration is a reason for the beginning of phase change. At present, the mechanism of this phenomenon is not clear enough, it can be considered due to the role of mechanical vibration in the solution appeared in the concentration of fluctuations, resulting in high over saturation region, and in which the nucleus began to generate. Solution stirring usually promotes crystal formation, but it is necessary to take into account the range of supersaturation in order to talk about the effect of agitation [42]. For example, where the solution is in the first metastable zone, agitation should not affect nucleation as long as the agitation does not bring the active center of the crystals into the solution. Stirring in the second zone will accelerate nucleation.

For secondary nucleation, stirring plays an extremely important role. The number of collisions and the impact of the impact itself can be increased, which are formed on the secondary nucleation has a great impact.

\section{4) Various fields}

Sobrnikov ${ }^{[46]}$ and many other researchers have studied the effect of electric field on nucleation in solution, both studying the constant field and studying the AC field. The results show that the magnitude of the electric field depends on its frequency and intensity. At present its mechanism of action is not clear. In addition, magnetic field, radioactive ray and roenton ray have an effect on nucleation rate, but less research on it now has not yet attracted the attention of researchers $^{[46]}$.

The nuclei appear before the molecular association, the solution from the steady state to the unstable state of the process, these associations gradually began to increase, and finally the formation of new particles. So the nucleus is not the beginning of the emergence of it takes some time, at the beginning, the largest nuclear particles into the nucleus, followed by smaller particles, these particles are gradually growing up. The relationship between nucleation rate and time is shown in Figure 2.5 $5^{[43]}$

And the induction period of nucleation refers to the time from the beginning to the generation of nuclei at a certain temperature, which is related to the nucleation rate ${ }^{[42]}$

$$
\tau \propto J^{-1}
$$

The nucleation rate is proportional to the reciprocal of the induction period, and the shorter the induction period, the greater the nucleation rate.

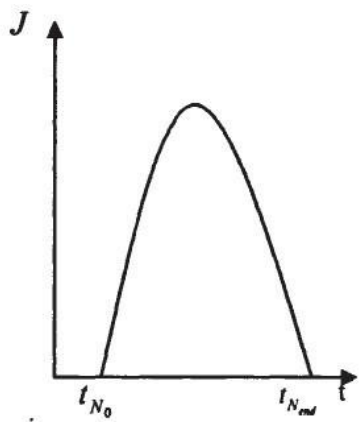

Figure 2.5 The relationship between nucleation rate and time

Fig. 2.5 Relation between nucleation rate and time

\subsection{Summary}

This chapter mainly introduces the thermodynamics principle of crystallization process, describes several different ways of nucleation, calculates some nucleation critical parameters of these nucleation methods, and analyzes the influencing factors of nucleation mode. Finally, the introduction for definition of nucleation rate and its influencing factors. For the crystal nucleation and growth of theoretical knowledge to prepare.

\section{Conclusions}

In this paper, the stability of KDP solution was first studied. The effects of different dibasic (EDTA $+\mathrm{kcl}$ ) concentration on the solubility of KDP and the width of metastable zone of saturated solution were discussed. At the same time, the effects of different doping and different supersaturation KDP supersaturated solution, and the nucleation thermodynamics parameters were calculated by combining the classical homogeneous nucleation theory. In the case of no rotation, the KDP crystal growth under different doping concentration and different saturation (100) plane was studied, and the growth mechanism of (100) plane was determined. Finally, the KDP was grown by chemical etching method under different conditions. The crystal (100) was etched and the dislocation distribution of the etched surface was observed with an optical microscope. The main conclusions are as follows:

(1) The experimental results show that the doubling has a significant effect on the solubility of KDP. With the increase of doping concentration, the solubility of KDP is obviously decreased, and the width of metastable zone increases with the increase of doping concentration. Sex has also been improved accordingly.

(2)According to the classical uniform nucleation theory, the KDP supersaturated solution into the nucleation of what research. The nucleation induction period of KDP solution at different temperature, different supersaturation and different doping concentration was determined by experiment. The results show that the nucleation induction period of KDP supersaturated solution increases with the 
supersaturation ratio, and when KDP solution supersaturation ratio $\mathrm{S} \geq 1.15$, the nucleation method is uniform Nuclear, $\mathrm{S}<1.15$, the nonuniform nucleation dominates.

(3) The nucleation thermodynamics parameters were calculated by the classical uniform nucleation theory. It can be seen from the calculated results that the doping can increase the critical nucleation energy and the solid-liquid interface tension, which makes the stability of the solution system improve.

(4)Through the calculation of the surface entropy factor, the micro-growth mechanism of KDP crystal under the experimental conditions was determined as continuous growth mode.

(5) By measuring the growth rate of KDP crystal (100) surface grown under different growth conditions, it can be found that doping can increase the growth rate of (100) surface to a certain extent.

(6) The growth mechanism of KDP crystal (100) plane was determined as the two-dimensional nucleation growth mechanism. The step edge energy of two-dimensional nucleation was calculated, and the doping was analyzed microscopically. To a certain extent, can improve the growth rate of (100) surface, which is consistent with the experimental results.

(7) It can be found that the dislocation density increases with the increase of saturation when the dislocation of the KDP crystal (100) plane is increased. Under the same supersaturation, the dislocation density increases with the increase of the dopant concentration, (1 mol\% $\mathrm{kcl}$ and $0.01 \mathrm{~mol} \%$ EDTA) can make the dislocation density small (even less than undoped), but also the solution has a certain degree of stability.

Although the research results of KDP solution stability, crystallization thermodynamics and dislocation density distribution have made some research results, but there are still some limitations in the research, the following is to be further carried out and improve the work:

(1) Since the stability of the solution after doping is studied in this paper, it is not perfect to study the change of crystal optical quality due to the change of stability. Therefore, in studying the stability of solution, we can use some special the equipment observes the optical properties of the crystal after the solution stability changes, such as the transmittance of the crystal, the observation of the crystal scattering particles, and so on.

(2) In the process of crystal growth, due to the use of this experiment does not rotate and the crystal placed in the bottom of the growth of the crystal, so that in the crystal growth process will be in the crystal surface of crystal or crystal appear on the crystal growth is extremely unfavorable. It is necessary to improve the crystal growth apparatus so that the crystal grows better.

\section{REFERENCES}

[1] Frazer, Peninsky R.X-ray Analysis of the Ferroelectric Transition in KH2PO4 [J]. Acta. Copperogr, 1953, 6: 273.

[2] Bacon G E, Pease R S. A Neutron Diffraction Study of Potassium Dihydrogen Phosphate by Fourier Synthesis [J]. Proc. Roy. Soc, Lond., 1953, 220: 397.

[3] Hartman P. The Morphology of Zircon and Potassium Dibydrogen Phosphate in Relation to the Crystal Structure [J]. Acta. Cryst, 1956, 9: 721.

[4] Zhang Kehua, Wang Ximin. Nonlinear optical crystal materials science [M]. Science Press, 1996,86-150.

[5] Blue letter. Laser technology. Science Press [M]. 2000.

[6] Wang Ganchang, Yuan Zhanyang. Inertial confinement fusion [M]. Anhui Education Press, 1996.

[7] Su Genbo, Zeng Jinbo, He Youping et al. Application of Large Cross Section Crystals in Laser Fusion [J]. Acta Phyllosulfurica Sinica, 1997,25: 717-719.

[8] Zhang Kehua, Wang Ximin. Nonlinear optical crystal materials science [M]. Science Press, 1994.

[9] Yao Lianzeng. Crystal growth base [M]. Hefei: China University of Science and Technology Press, 1995.

[10] Min Nai. The physical basis of crystal growth [M]. Shanghai: Shanghai Science and Technology Press, 1982: 345.

[11] Zhang Lichuan, Chen Qiyuan, etc. Conductivity method for the study of supersaturated sodium aluminate solution homogeneous nucleation process, 2008,26 (6): 1111-1114.

[12] A.E. Nielsen, O. Sohnel. Homogeneous nucleation of droplets and interfacial tension in the liquid system methanol-water-tribromomethane [J]. Journal of Crystal Growth, 1971, 8: 1-7.

[13] Chen J, et al. Nucleation of electrohydraulic crystal hydrogen rubbenate supersaturated solution. Journal of Synthetic Crystals, 2000,29 (4): 376-380 (in Chinese with English abstract)

[14] Liu B, et al. Study on primary nucleation of solution by induction period. Journal of Tianjin University, 2003,36 (5): 553-556.

[15] YANG Liu, CHEN Qi-yuan, YIN Zhou-lan et al. Study on the primary nucleation process of supersaturated sodium aluminate solution by induction period [J]. Nonferrous Metals, 2008 (4).

[16] M.S. Joshi, A. V. Antony. Nucleation in supersaturated potassium dihydrogen orthophosphate solutions [J]. Journal of Crystal Growth, 1979, 46 (1): 7-9.

[17] K. Wojciechowski, W. Kibalczyc. Light scattering study of KH2PO4 and baso4 nucleation process [J]. Journal of Crystal Growth, 1986, 76 (2): 379-382. 